Ebisu Ebisu

Études japonaises Études japonaises

49 | printemps-été 2013

De chose en fait : la question du milieu

\title{
Bernard FRANK, Démons et jardins. Aspect de la civilisation du Japon ancien
}

Paris, Collège de France, Institut des hautes études japonaises, 2011, $342 \mathrm{p}$.

Sylvie Brosseau

\section{CpenEdition}

1 Journals

Édition électronique

URL : http://journals.openedition.org/ebisu/831

DOI : 10.4000/ebisu.831

ISSN : 2189-1893

Éditeur

Institut français de recherche sur le Japon à la Maison franco-japonaise (UMIFRE 19 MEAE-CNRS)

Édition imprimée

Date de publication : 1 avril 2013

Pagination : 167-171

ISSN : 1340-3656

\section{Référence électronique}

Sylvie Brosseau, «Bernard FRAnk, Démons et jardins. Aspect de la civilisation du Japon ancien », Ebisu [En ligne], 49 | printemps-été 2013, mis en ligne le 25 mars 2014, consulté le 22 septembre 2020. URL: http://journals.openedition.org/ebisu/831 ; DOI : https://doi.org/10.4000/ebisu.831

Ce document a été généré automatiquement le 22 septembre 2020.

(c) Institut français de recherche sur le Japon à la Maison franco-japonaise 


\section{Bernard FRANK, Démons et jardins. Aspect de la civilisation du Japon ancien}

Paris, Collège de France, Institut des hautes études japonaises, 2011, $342 \mathrm{p}$.

Sylvie Brosseau

\section{RÉFÉRENCE}

Bernard FRANK, Démons et jardins. Aspect de la civilisation du Japon ancien, Paris, Collège de France, Institut des hautes études japonaises, 2011, 342 p.

1 Cet ouvrage réunit un ensemble de textes, souvent introuvables ou encore inédits, écrits entre 1953 et 1994 par Bernard Frank (1927-1996), premier titulaire de la chaire de Civilisation japonaise au Collège de France en 1979, d'abord chercheur érudit qui a étudié conjointement la religion et la littérature. Seize textes ont été rassemblés par un utile travail éditorial resté anonyme sous un titre qui annonce les thèmes abordés : d'abord, « les démons dans la littérature et l'art japonais anciens »; puis, " Minamoto no Tōru, et son jardin du Kawara no in ». Ces deux parties sont constituées par les résumés de deux séries de cours donnés à l'École pratique des hautes études entre 1970 et 1979 , complétés par des traductions (de contes comme de textes poétiques), des articles, des textes de conférences, des annexes sur des sujets liés tels que, par exemple, la notion double d'ukiyo, 憂世 monde d'affliction ou 浮世 monde flottant suivant la graphie utilisée, ou bien la figure de l'île errante Ukishima 浮島.

2 Ces deux sous-ensembles peuvent sembler étrangers l'un à l'autre mais plusieurs fils les relient. D'abord, le cadre temporel considéré est celui du Japon ancien, principalement de l'époque de Heian, sujet d'étude et même d'une certaine forme de vénération de la part de l'auteur. Autres liens, des récits démoniaques apparaissent dans les deux parties, la première étant centrée sur les diverses formes et apparitions des oni 鬼, démons; et un personnage, le ministre Minamoto no Tōru 源融(822-895), prince écarté du lignage impérial, au destin indissociable du jardin qu'il a créé, est évoqué dans la 
première partie avant d'être au cœur de la seconde. Au fil des textes, l'auteur nous fait suivre l'histoire de ce jardin unique et extraordinaire jusqu'à sa disparition matérielle, puis nous fait observer les diverses réminiscences que ce lieu suscita. Outre ces liens, on peut discerner un leitmotiv thématique à l'ensemble de cet ouvrage, celui de la transformation perpétuelle de tout être et de toute chose, fictionnels ou réels, dont d'ailleurs les limites se brouillent.

3 L'auteur a rassemblé la majeure partie de ce que l'on sait sur Minamoto no Tōru, sur sa façon de penser, ses références esthétiques, ses préoccupations morales et religieuses, ses attentes. Tōru, que Bernard Frank qualifie d'«esprit audacieux et novateur", d'«original de haut vol», de "génie excentrique et inspirateur", créa dans sa résidence du Kawara no in 河原院 à Kyoto un jardin parmi les plus vastes de l'époque (4 chō 町, soit un peu moins de 4 hectares). Celui-ci représentait le site de Shiogama 塩 釜 près de Matsushima 松島, situé dans le nord-est du Japon, donc loin de la capitale mais qui exerçait un attrait fascinant sur les lettrés de la cour. Ce site était célèbre, à travers la poésie, à la fois pour la beauté unique de son paysage maritime, baie parsemée d'îlots rocheux aux pins tortueux, ainsi que pour son activité d'extraction du sel. Cette pratique riche de symboles et indissociable du site s'effectuait (et s'effectue toujours) à partir d'algues laminaires réduites en cendre, suivant un procédé long et complexe que Bernard Frank détaille. Dans son jardin, Tōru poussa l'évocation jusqu'à faire remplir l'étang avec de l'eau de mer pour pouvoir en extraire le sel comme à Shiogama, dans les fumées et les vapeurs. Versé dans la culture de la Chine, il connaissait sans aucun doute les descriptions chinoises des palais qui rassemblaient en miniatures les sites fameux de l'Empire ainsi que des composants rares de la nature, manière de s'approprier par la maîtrise d'un microcosme les forces du monde, dont celles qui donnent la longévité, voire l'immortalité. Au temps de sa splendeur, les poètes les plus éminents fréquentaient le Kawara no in où Tōru, lui-même poète, jouait le rôle de mécène pour un cénacle en quête de l'esprit du füryū 風流, style de vie aristocratique qui tend vers une unité esthétique et morale inspirée du taoïsme. Bernard Frank détaille cette voie du fūryū, avant tout poétique et esthétique, mais dont les significations religieuses ou philosophiques ne peuvent être complètement écartées.

4 Après la disparition du maître des lieux en 895 , la propriété ne fut pas immédiatement abandonnée mais, devenue résidence impériale fréquentée ponctuellement, plusieurs rencontres poétiques s'y déroulèrent encore, puis diverses modifications la transformèrent en lieu de culte bouddhique. L'ancien palais devint un ermitage dévolu à la nostalgie, dans lequel des poètes venaient méditer sur l'impermanence de ce monde et ressourcer leur idéal de lettrés en guettant les signes de l'automne dans la nature et les marques du temps dans le déclin des choses. Finalement, le jardin et les bâtiments furent irrémédiablement ruinés par un typhon en 979, l'abandon semble complet vers l'an 1000, et des incendies achevèrent de ravager ce qui restait. Mais déjà, le lieu n'avait plus qu'une mauvaise réputation due aux apparitions du spectre de Tōru, d'abord en tant que propriétaire irascible, accroché à son bien donc aux apparences, qui essayait de chasser les visiteurs intrus. Bernard Frank note avec un humour teinté d'ironie que le maître des lieux a manifestement raté l'accession à l'état d'Immortel qu'il visait. Puis, à propos du Kawara no in surgissent des histoires démoniaques carrément épouvantables et sanglantes. En guise d'épilogue à ces divers épisodes, l'auteur tire une déduction concrète sur ce que révèlent les fantômes: tant que les 
rancœurs, les ressentiments accumulés dans le passé ne sont pas complètement éteints, ceux-ci continuent à bel et bien perdurer dans le présent.

5 Les mauvais souvenirs à leur tour évanouis, le jardin du Kawara no in continua à inspirer des poèmes et d'autres formes de jardins. Bernard Frank cite entre autres le sanctuaire Itsukushima 厳島神社 à Miyajima 宮島 fondé au XII siècle par Taira no Kiyomori 平清盛, qui en représenterait une forme des plus abouties d'après un auteur japonais cité. Beaucoup plus tard, on pourrait ajouter les jardins de l'époque d'Edo comme le Hama-rikyū 浜離宮, proche du littoral de la cité d'Edo, jardin d'agrément qui a intégré et esthétisé le rythme de la marée dans son agencement, tour à tour dévoilant et recouvrant les rochers dans l'étang, et qui évoquait aussi Shiogama par des huttes de sauniers en activité, référence très explicite au Kawara no in. En définitive, le site de Shiogama finit par entraîner très naturellement l'évocation du palais et de la personnalité de Tōru. Par exemple, le destin de cet homme suggéra à Zeami 世阿弥, au $\mathrm{XV}^{\mathrm{e}}$ siècle, une pièce de nō où, dans l'éclat de la pleine lune, son esprit descend danser une longue transe durant le sommeil d'un moine voyageur parvenu à ce site fameux. Les textes réunis dans ce volume nous permettent donc de reconstituer les différentes phases du déclin d'un palais, après une apogée unique et prodigieuse jusqu'à sa disparition totale, suivant une série de transformations riches de significations. Ces métamorphoses donnent à percevoir également de quelle manière se manifeste le retour du génie du lieu, transposé, et finalement immortalisé, sous diverses formes. La thématique du retour est un autre fil conducteur à l'ensemble des textes réunis.

6 À travers ces écrits, comme dans toute son œuvre, Bernard Frank n'a cessé de replacer les représentations culturelles des Japonais à l'intérieur d'un environnement local pénétré de divers flux venus du continent. Les apports spirituels et intellectuels d'origines chinoises ou indiennes, tels que les idéaux taoïstes, la voie du yin et du yang, et bien sûr le bouddhisme, nous révèlent les liens profonds qui unissent l'Inde, la Chine et le Japon et nous rappellent que la civilisation japonaise n'est pas un isolat historique et culturel mais appartient à l'ensemble des cultures d'Extrême-Orient. L'érudition de l'auteur au sujet des cultures et des langues de la Chine et de l'Inde enrichit une œuvre qui reste accessible car servie par une écriture qui évite toute forme de pédantisme ou de jargon, mais au contraire, reste très alerte, alternant des tonalités diverses, de la touche ironique et enjouée à la réflexion concise et profonde. De plus, le travail de Bernard Frank a la spécificité de joindre, dans la mesure du possible, aux résultats des études philologiques les plus approfondies, les fruits de l'observation et de l'expérience vécue. Il ajoute à la science érudite une dimension ethnographique et sensible tirée de sa fréquentation du terrain et de la réalité concrète. Sans tomber dans l'anecdote, il montre une forme d'empathie pour les personnages rencontrés lors de ses recherches, comme par exemple Minamoto no Shitagau 源順, lettré profondément malheureux, peu reconnu et peu aidé au cours de sa vie, au $\mathrm{x}^{\mathrm{e}}$ siècle, mais qu'il considère comme un poète des plus créatifs de toute l'histoire du Japon. Quant à Minamoto no Tōru, il est qualifié de "personnalité des plus attachantes » de son époque. À travers le temps et l'espace, il s'agit bien de rencontres avec des individualités humaines, non pas seulement d'objets d'étude.

7 Tous ceux qui cherchent à mieux comprendre la perception des paysages et les caractéristiques des jardins du Japon, à en connaître certains ressorts philosophiques, à en préciser des éléments symboliques, religieux, littéraires, seront intéressés par la lecture de ce livre qui donne accès à des sources anciennes d'une approche difficile. Il 
permet aussi de découvrir ou de retrouver la grandeur et la générosité d'une œuvre indissociable d'une quête personnelle.

\section{AUTEURS}

SYLVIE BROSSEAU

Université Waseda 